\title{
Decentralized Sliding Mode Control for Uncertain Discrete-Time Large-Scale Systems: An LMI Approach
}

\author{
Ahmadreza Argha, Li Li, Steven W. Su${ }^{\star}$ and Hung Nguyen
}

\begin{abstract}
In this paper, a decentralized discrete-time sliding mode control is designed for the uncertain large-scale systems. Firstly, a decentralized sliding surface is developed for the largescale discrete-time systems including uncertainty and exogenous disturbance. Then, a decentralized sliding mode controller is designed for the underlying systems. An LMI approach is deployed to develop a new framework to design the decentralized sliding mode controller which can stabilize the underlying uncertain large-scale system. The ultimate boundedness of the state and sliding function of the underlying closed-loop system is studied accordingly. Illustrative examples are presented to show the effectiveness of the proposed controllers.
\end{abstract}

\section{INTRODUCTION}

Discrete-time sliding mode control (DSMC) has been firstly proposed in the mid 80s [1]. This idea was followed by a vast variety of publications [2] - [5]. It is well-known that the finite sampling rate in the discrete-time systems would lead to the fact that the state trajectories could not remain on the ideal sliding surface. Indeed, the state trajectories of the discrete-time system would move within a boundary layer around the predefined sliding surface known as quasi-sliding mode band [6].

A wide range of the early DSMC investigations have considered the possibility of creating a discrete-time counterpart to the continuous-time reachability condition [7], [8], [6]. However, it is argued in the literature that DSMC, unlike continuous-time SMC (CSMC), does not necessarily need to include a variable structure discontinuous control (VSDC) strategy [10], [9], [11]. References [10] and [9] have shown that using the so-called linear control can guarantee that the state trajectories remain within a neighbourhood of the sliding surface in the presence of bounded matched uncertainty. According to the results given in [10], [9] the use of a switching function in the controller can not certainly improve the performance. More importantly, elimination of the discontinuous control part from the discrete-time sliding mode control law can eliminate the chattering issue [10], [9], [11].

An outstanding investigation implemented on SMC has been decentralized SMC for the large-scale interconnected systems; see [12] - [13] and the references therein. Other than recent works in [28] and [29], most of the literature is in decentralized CSMC, and, thus, the discrete-time decentralized SMC still requires more investigations. Furthermore,

Ahmadreza Argha, Li Li, Steven W. Su and Hung Nguyen are with Faculty of Engineering and Information Technology, University of Technology, Sydney, PO Box 123, Broadway, NSW 2007, Australia.

\{Ahmadreza.Argha, Li.Li, Steven.Su, Hung. Nguyen $\}$ @uts. edu. au references [28] and [29] consider a VSDC for the problem of decentralized DSMC, which according to [10], [9], [11], is non-beneficial in terms of performance. Indeed, these references assume that the sampling rate of the system is very high. With this assumption, the closed-loop system would behave more or less as a continuous-time system. This paper, basically, will follow different framework. Moreover, unlike [28] and [29], the underlying system, here, is assumed to involve unmatched uncertainties.

It should be noted that decentralized control, today, is a well-known field of study in the control community and it has a rich history in the literature [14]. As a common approach, a large-scale system is considered to be composed of interconnected sub-systems with a specific structure. One method is to deal with the interconnections between sub-systems as uncertainties of the overall system; see e.g. [15], [16]. As a result, the next step will be designing a decentralized controller which can tolerate the effect of interconnections to obtain a predetermined performance level. In order to achieve this goal, two imaginable approaches are either neglecting or using the interconnections in the controller design; see e.g. [17], [18]. In either method, interconnections are known as the factors that reduce the performance of the overall system. Alternatively, in practical applications, the interconnections or at least some of them might be known and provide useful information, especially for the large-scale systems with an insufficient number of decentralized controllers; see e.g. [19].

In this paper, we assume that the interconnections of the underlying system contain both known and uncertain parts. Using interconnections' information, an LMI method is exploited to design the decentralized sliding surface. To this end, imposing the block-diagonal structure on the LMI variable, a fully decentralized sliding surface is designed which can tolerate both interconnection influences and subsystems uncertainties. Accordingly, using the obtained decentralized sliding surface, a linear decentralized DSMC is proposed and the ultimate boundedness of the state and the sliding function of the underlying overall closed-loop system is analyzed. With the aim of comparison, and as an extension, a variable structure decentralized controller is also proposed and tested in this paper.

The rest of this paper is organized as follows: Section II describes the problem formulation and preliminaries. In Section III, the proposed method to design the decentralized DSMC is given, and in particular, the linear controller is investigated. Variable structure decentralized DSMC is the subject of Section IV. Efficiency of the proposed DSMC is studied by numerical examples in Section V. Finally, Section 
VI concludes this paper.

Notation: $\left[A_{i j}\right]_{h \times h}$ is a square block matrix with the block entries $A_{i j}, i=1, \cdots, h, j=1, \cdots, h \cdot \operatorname{diag}\left(A_{1}, \cdots, A_{h}\right)$ is the block-diagonal matrix with diagonal entries $A_{i}, i=1, \cdots, h$. $\|v\|$ is the $\ell_{2}$-norm of vector $v$. The function $s g n$ denotes the well-known signum function.

\section{Problem Formulation And Preliminaries}

Consider a large scale system including $h$ sub-systems,

$$
\begin{gathered}
\Sigma_{i}: x_{i}(k+1)=\left[A_{i i}+\Delta A_{i i}(k)\right] x_{i}(k)+B_{i}\left[u_{i}(k)+f_{i}(k)\right] \\
+\sum_{\substack{j=1 \\
i \neq j}}^{h}\left[A_{i j}+\Delta A_{i j}(k)\right] x_{j}(k), i=1, \cdots, h,
\end{gathered}
$$

where $x_{i} \in \mathbb{R}^{n_{i}}$ and $u_{i} \in \mathbb{R}^{m_{i}}$ are the state vector and control input vector of the $i$ th sub-system, respectively. The matrices in (1) are constant and of appropriate dimensions. The term $\Delta A_{i i}(k)$ denotes the uncertainty of $i$ th sub-system and $\sum_{\substack{j=1 \\ i \neq j}}^{h} A_{i j} x_{j}(k), \sum_{\substack{j=1 \\ i \neq j}}^{h} \Delta A_{i j}(k) x_{j}(k)$ are, respectively, a known interconnection and an uncertain interconnection of the $i$ th sub-system. $f_{i}(k)$ implies the matched external disturbances of the $i$ th sub-system with known bound.

Define

$$
x:=\left[\begin{array}{c}
x_{1} \\
\vdots \\
x_{h}
\end{array}\right], u:=\left[\begin{array}{c}
u_{1} \\
\vdots \\
u_{h}
\end{array}\right], f(k):=\left[\begin{array}{c}
f_{1}(k) \\
\vdots \\
f_{h}(k)
\end{array}\right],
$$

and

$$
A:=\left[A_{i j}\right]_{h \times h}, \Delta A:=\left[\Delta A_{i j}\right]_{h \times h}, B:=\operatorname{diag}\left(B_{1}, \cdots, B_{h}\right) .
$$

Besides, the uncertainty matrix for compact system is assumed to be as follows,

$$
\Delta A(k):=\left[\begin{array}{c}
M_{1} \\
\vdots \\
M_{h}
\end{array}\right] R(k)\left[\begin{array}{lll}
N_{1} & \cdots & N_{h}
\end{array}\right],
$$

where

$$
\|R(k)\|<1, \forall k \geq 0
$$

Defining

$$
M:=\left[\begin{array}{c}
M_{1} \\
\vdots \\
M_{h}
\end{array}\right], N:=\left[\begin{array}{lll}
N_{1} & \cdots & N_{h}
\end{array}\right],
$$

one can write

$$
\Delta A(k):=M R(k) N .
$$

Using (2) and (3) the compact form of the sub-systems in (1) can be written as

$$
\Sigma: x(k+1)=[A+\Delta A(k)] x(k)+B[u(k)+f(k)] .
$$

The following lemmas are useful in the sequel.
Lemma 1 ( [21]): Let $E, F(k)$ and $G$ be real matrices of appropriate dimensions with $F^{T}(k) F(k)<I, \forall k \geq 0$, then, for any scalar $\varepsilon>0$, we have

$$
E F(k) G+G^{T} F^{T}(k) E^{T} \leq \varepsilon E E^{T}+\varepsilon^{-1} G^{T} G .
$$

Lemma 2 (Schur Complement [20]): The following linear matrix inequality,

$$
\left[\begin{array}{ll}
Q_{1} & H \\
H^{T} & Q_{2}
\end{array}\right]>0
$$

is equivalent to $Q_{2}>0, Q_{1}-H Q_{2}^{-1} H^{T}>0$, where $Q_{1}^{T}=Q_{1}$, $Q_{2}^{T}=Q_{2}$ and $H$ is a matrix with appropriate dimension.

Remark 1: In this paper, it is assumed that the uncertain parameters of the subsystems and the interconnections between sub-systems are similar, which results in the simplification of the analysis. However, corresponding decentralized SMC for the general form of uncertainties will be considered in the future research.

\section{DECENTRALIZED DISCRETE-TIME SLIDING MODE CONTROL}

Consider the following decentralized linear discrete-time sliding function:

$$
\sigma_{x}(k)=S x(k),
$$

where $\sigma_{x}(k):=\operatorname{col}\left(\sigma_{x_{1}}(k), \cdots, \sigma_{x_{h}}(k)\right)$ and the block diagonal matrix $S:=\operatorname{diag}\left(S_{1}, \cdots, S_{h}\right)$ will be designed later such that $S B$ is nonsingular.

During the ideal sliding motion the sliding function satisfies:

$$
\sigma_{x_{i}}(k+1)=\sigma_{x_{i}}(k)=0, \quad \forall k>k_{s},
$$

where $k_{s}>0$ denotes the time that sliding motion starts. However, one may obtain from (8) and (9) that

$$
\sigma_{x}(k+1)=S(A+\Delta A) x(k)+S B[u(k)+f(k)] .
$$

Assume $f_{i}(k)=\operatorname{col}\left(f_{i 1}, \cdots, f_{i m_{i}}\right)$, and

$$
f_{i j}^{l} \leq f_{i j} \leq f_{i j}^{u}, \quad j=1, \cdots, m_{i},
$$

also define

$$
\begin{aligned}
f_{i j}^{+} & =\frac{f_{i j}^{u}+f_{i j}^{l}}{2}, \quad f_{i j}^{-}=\frac{f_{i j}^{u}-f_{i j}^{l}}{2}, \\
\mathscr{F}_{i}^{+} & =\operatorname{col}\left(f_{i 1}^{+}, \cdots, f_{i m_{i}}^{+}\right), \quad \mathscr{F}_{i}^{-}=\operatorname{diag}\left(f_{i 1}^{-}, \cdots, f_{i m_{i}}^{-}\right) \\
\mathscr{F}_{i}^{c} & =\operatorname{col}\left(f_{i 1}^{-}, \cdots, f_{i m_{i}}^{-}\right),
\end{aligned}
$$

and

$$
A_{D}:=\operatorname{diag}\left(A_{11}, \cdots, A_{h h}\right) .
$$

Now, the following compact control law is proposed,

$$
u(k)=-(S B)^{-1} S A_{D} x(k)-\vartheta(k),
$$

where $\vartheta(k)=\operatorname{col}\left(\vartheta_{1}(k), \cdots, \vartheta_{h}(k)\right)$, in which $\vartheta_{i}(k) \in \mathbb{R}^{m_{i}}$, denotes the approximation of disturbance $f(k)$ which may be used in the controller to compensate the bad effect of disturbance on the ultimate bound on the system state trajectories. $\vartheta(k)$ can be also regarded as the feedforward 
control, in addition to the linear controller. It is assumed that the component $\vartheta(k)$ is bounded and, hence,

$$
\|f(k)-\vartheta(k)\| \leq \tau_{i}\left\|\mathscr{F}^{c}\right\|,
$$

where $\tau$ is a positive scalar, and $\mathscr{F}^{c}=\operatorname{col}\left(\mathscr{F}_{1}^{c}, \cdots, \mathscr{F}_{h}^{c}\right)$; see (12), (13). Some choices for $\vartheta_{i}(k)$ are presented later in the next section.

Remark 2: Note that, here, to construct the control law (15), only the upper and lower bounds on matched disturbance are used; see (12), (13). Although it can be seen in the literature that the term $\Delta A x(k)$ or $\Delta A_{D} x(k)$, where $\Delta A_{D}(k):=\operatorname{diag}\left(\Delta A_{11}, \cdots, \Delta A_{h h}\right)$, is assumed to be bounded; see e.g. [24], [2] and [25], this assumption implies the boundedness of system states, thus, the stability of the system from the beginning. Hence, in this paper, we construct the nonlinear part of the controller by using only the external disturbance information.

The sequel of this section, aims to consider the stability of the system (8) using the controller (15). As a result of applying the controller (15) to the system (8), it is seen that

$$
x(k+1)=(A+\Delta A-\hat{A}) x(k)+B[f(k)-\vartheta(k)],
$$

where $\hat{A}=B(S B)^{-1} S A_{D}$. Furthermore, it can be found that

$$
\sigma_{x}(k+1)=S\left(A-A_{D}+\Delta A\right) x(k)+(S B)[f(k)-\vartheta(k)] .
$$

The following lemmas are given to consider the boundedness of the state of the system (17).

Lemma 3 ( [26]): Let $V(\zeta(k))$ be a Lyapunov candidate function. In the case that there exist real scalars $v \geq 0, \alpha>0$, $\beta>0$ and $0<\rho<1$ such that

$$
\alpha\|\zeta(k)\|^{2} \leq V(\zeta(k)) \leq \beta\|\zeta(k)\|^{2},
$$

and

$$
V(\zeta(k+1))-V(\zeta(k)) \leq v-\rho V(\zeta(k)),
$$

then $\zeta(k)$ will satisfy

$$
\|\zeta(k)\|^{2} \leq \frac{\beta}{\alpha}\|\zeta(0)\|^{2}(1-\rho)^{k}+\frac{\nu}{\alpha \rho} .
$$

Lemma 4: For any symmetric block diagonal matrix $P>0$ and any full column rank block diagonal matrix $B$, we have $P B\left(B^{T} P B\right)^{-1} B^{T} P \leq P$.

Proof: It can easily be proved by

$$
\left[I-B\left(B^{T} P B\right)^{-1} B^{T} P\right]^{T} P\left[I-B\left(B^{T} P B\right)^{-1} B^{T} P\right] \geq 0 .
$$

Theorem 1: The compact form of the local control law (15) can drive the state of the overall system (8) into a boundary layer around the ideal sliding surface (9) and, in addition, the system state trajectories are ultimately bounded if there exist a symmetric block diagonal matrix $\bar{P}>0$, where $\bar{P}=\operatorname{diag}\left(\bar{P}_{1}, \cdots, \bar{P}_{h}\right)$, in which $\bar{P}_{i} \in \mathbb{R}^{n_{i} \times n_{i}}$, matrices $X, Y$ and also scalars $\varepsilon>0$ and $\bar{\eta}>0$ satisfying the LMI in (19), shown at the top of the next page, where $S=B^{T} \bar{P}^{-1}$.

Proof: Define

$$
V(\zeta(k))=x^{T}(k) P x(k)+\sigma_{x}^{T}(k)(S B)^{-1} \sigma_{x}(k),
$$

where $P=\operatorname{diag}\left(P_{1}, \cdots, P_{h}\right)$, in which $P_{i} \in \mathbb{R}^{n_{i} \times n_{i}}$ is a symmetric block diagonal and positive definite matrix, $S=B^{T} P$ and $\zeta(k)=\left[\begin{array}{ll}x^{T}(k) & \sigma_{x}^{T}(k)\end{array}\right]^{T}$. Thus, we can write

$$
\begin{aligned}
\Delta V(\zeta(k))= & V(\zeta(k+1))-V(\zeta(k)) \\
= & x^{T}(k+1) \operatorname{Px}(k+1)+\sigma_{x}^{T}(k+1)(S B)^{-1} \sigma_{x}(k+1) \\
& -x^{T}(k) \operatorname{Px}(k)-\sigma_{x}^{T}(k)(S B)^{-1} \sigma_{x}(k) .
\end{aligned}
$$

It can be shown that

$$
\begin{aligned}
& x^{T}(k+1) P x(k+1) \\
= & x^{T}(k)\left[A+\Delta A-B(S B)^{-1} S(A+\Delta A)+B(S B)^{-1} S\right. \\
& \left.\times\left(A-A_{D}+\Delta A\right)\right]^{T} P\left[A+\Delta A-B(S B)^{-1} S(A+\Delta A)\right. \\
& \left.+B(S B)^{-1} S\left(A-A_{D}+\Delta A\right)\right] x(k) \\
& +2 x^{T}(k)\left(A-A_{D}+\Delta A\right)^{T} S^{T}[f(k)-\vartheta(k)] \\
& +[f(k)-\vartheta(k)]^{T}(S B)[f(k)-\vartheta(k)] \\
= & x^{T}(k)\left[A+\Delta A-B(S B)^{-1} S(A+\Delta A)\right]^{T} \\
\times & P\left[A+\Delta A-B(S B)^{-1} S(A+\Delta A)\right] x(k) \\
+ & x^{T}(k)\left(A-A_{D}+\Delta A\right)^{T} S^{T}(S B)^{-1} S\left(A-A_{D}+\Delta A\right) x(k) \\
+ & 2 x^{T}(k)\left(A-A_{D}+\Delta A\right)^{T} S^{T}[f(k)-\vartheta(k)] \\
+ & {[f(k)-\vartheta(k)]^{T}(S B)[f(k)-\vartheta(k)] . }
\end{aligned}
$$

Also,

$$
\begin{aligned}
& \sigma_{x}^{T}(k+1)(S B)^{-1} \sigma_{x}(k+1) \\
= & x^{T}(k)\left(A-A_{D}+\Delta A\right)^{T} S^{T}(S B)^{-1} S\left(A-A_{D}+\Delta A\right) x(k) \\
& +[f(k)-\vartheta(k)]^{T}(S B)[f(k)-\vartheta(k)] \\
& +2 x^{T}(k)\left(A-A_{D}+\Delta A\right)^{T} S^{T}[f(k)-\vartheta(k)] .
\end{aligned}
$$

Besides, note that

$$
\begin{aligned}
& \left.2 x^{T}(k)\left(A-A_{D}+\Delta A\right)^{T} S^{T}[f(k)-\vartheta(k)]\right] \\
\leq & x^{T}(k)\left(A-A_{D}+\Delta A\right)^{T} S^{T}(S B)^{-1} S\left(A-A_{D}+\Delta A\right) x(k) \\
& +[f(k)-\vartheta(k)]^{T}(S B)[f(k)-\vartheta(k)] .
\end{aligned}
$$

Now, using (22), (23), (24), (16), we have

$$
\begin{aligned}
& \Delta V(\zeta(k)) \\
\leq & x^{T}(k)\left[(A+\Delta A(k))^{T} P(A+\Delta A(k))\right. \\
& -(A+\Delta A(k))^{T} P B\left(B^{T} P B\right)^{-1} B^{T} P(A+\Delta A(k))-P \\
& -P B\left(B^{T} P B\right)^{-1} B^{T} P+4\left(A-A_{D}+\Delta A\right)^{T} P B\left(B^{T} P B\right)^{-1} B^{T} P \\
& \left.\times\left(A-A_{D}+\Delta A\right)\right] x(k)+4[f(k)-\vartheta(k)]^{T}(S B)[f(k)-\vartheta(k)] .
\end{aligned}
$$

According to Lemma 4 and (16), it can be noted that

$$
\begin{aligned}
& \Delta V(\zeta(k)) \\
\leq & x^{T}(k)\left[(A+\Delta A(k))^{T} P(A+\Delta A(k))\right. \\
& -(A+\Delta A(k))^{T} P B\left(B^{T} P B\right)^{-1} B^{T} P(A+\Delta A(k))-P \\
& -P B\left(B^{T} P B\right)^{-1} B^{T} P+4\left(A-A_{D}+\Delta A\right)^{T} P \\
& \left.\times\left(A-A_{D}+\Delta A\right)\right] x(k)+\gamma \\
\triangleq & x^{T}(k) \Theta x(k)+\gamma,
\end{aligned}
$$




$$
\left[\begin{array}{cccccc}
-\bar{P}+Y^{T} B^{T}+B Y & (A \bar{P}+B X)^{T} & Y^{T} B^{T} & 2 \bar{P}\left(A-A_{D}\right)^{T} & \bar{P} & \bar{P} N^{T} \\
A \bar{P}+B X & -\bar{P}+\varepsilon M M^{T} & 0 & 2 \varepsilon M M^{T} & 0 & 0 \\
B Y & 0 & -\bar{P} & 0 & 0 & 0 \\
2\left(A-A_{D}\right) \bar{P} & 2 \varepsilon M M^{T} & 0 & -\bar{P}+4 \varepsilon M M^{T} & 0 & 0 \\
\bar{P} & 0 & 0 & 0 & -\bar{\eta} I & 0 \\
N \bar{P} & 0 & 0 & 0 & 0 & -\varepsilon I
\end{array}\right]<0
$$

where $\gamma=4 \tau^{2}\|S B\|\left\|\mathscr{F}^{c}\right\|^{2}$, and

$$
\begin{aligned}
\Theta:= & (A+\Delta A(k))^{T} P(A+\Delta A(k)) \\
& -(A+\Delta A(k))^{T} P B\left(B^{T} P B\right)^{-1} B^{T} P(A+\Delta A(k)) \\
& -P-P B\left(B^{T} P B\right)^{-1} B^{T} P \\
& +4\left(A-A_{D}+\Delta A\right)^{T} P\left(A-A_{D}+\Delta A\right) .
\end{aligned}
$$

Now, assuming that

$$
\Theta<-\eta I,
$$

we have

$$
\Delta V(\zeta(k)) \leq-\eta x^{T}(k) x(k)+\gamma,
$$

where $\eta>0$ is a scalar variable. To adapt the results of this theorem to Lemma 3, we have to do some manipulations. First, define

$$
\begin{aligned}
V(\zeta(k)) & =x^{T}(k)\left[P+P B\left(B^{T} P B\right)^{-1} B^{T} P\right] x(k) \\
& \triangleq x^{T}(k) M_{P} x(k) .
\end{aligned}
$$

Hence,

$$
\lambda_{\min }\left(M_{P}\right)\|x(k)\|^{2} \leq V(\zeta(k)) \leq \lambda_{\max }\left(M_{P}\right)\|x(k)\|^{2} .
$$

Furthermore, it is obvious that

$$
\begin{aligned}
\lambda_{\min }\left(\operatorname{diag}\left(P,(S B)^{-1}\right)\right) & \|\zeta(k)\|^{2} \leq V(\zeta(k)) \\
& \leq \lambda_{\max }\left(\operatorname{diag}\left(P,(S B)^{-1}\right)\right)\|\zeta(k)\|^{2} .
\end{aligned}
$$

Consequently, from (29) and (31), one can derive that

$$
\Delta V(\zeta(k)) \leq-\frac{\eta}{\lambda_{\max }\left(M_{P}\right)} V(\zeta(k))+\gamma .
$$

Note that from (28) and Lemma 4 it can easily be derived that $\eta I<M_{P}$, or equivalently, $\frac{\eta}{\left.\lambda_{\max } M_{P}\right)}<1$. Eventually, from Lemma 3 and (32), we conclude that

$$
\begin{aligned}
& \forall \varepsilon>0, \exists k^{\star}>0, \text { s.t. } \forall k>k^{\star}, \\
&\|\zeta(k)\|^{2} \leq \frac{\lambda_{\max }\left(M_{P}\right)}{\eta \lambda_{\min }\left(\operatorname{diag}\left(P,(S B)^{-1}\right)\right)} \gamma+\varepsilon .
\end{aligned}
$$

where $\varepsilon$ is a positive scalar. Now, let us consider the feasibility of $\Theta<-\eta I$ in (28), which is equivalent to that of

$$
\begin{aligned}
(A & +\Delta A(k))^{T} P(A+\Delta A(k))+\left[(A+\Delta A(k))^{T} P B\left(B^{T} P B\right)^{-1}\right. \\
& \left.+F^{T}\right]\left(B^{T} P B\right)\left[F+\left(B^{T} P B\right)^{-1} B^{T} P(A+\Delta A(k))\right] \\
& -(A+\Delta A(k))^{T} P B\left(B^{T} P B\right)^{-1} B^{T} P(A+\Delta A(k))-P \\
& -P B\left(B^{T} P B\right)^{-1} B^{T} P+\left[L+\left(B^{T} P B\right)^{-1} B^{T} P\right]^{T}\left(B^{T} P B\right) \\
& \times\left[L+\left(B^{T} P B\right)^{-1} B^{T} P\right]+4\left(A-A_{D}+\Delta A\right)^{T} P \\
& \times\left(A-A_{D}+\Delta A\right)<-\eta I
\end{aligned}
$$

where $F$ and $L$ are two auxiliary variables [22]. Hence, we derive

$$
\begin{aligned}
& (A+\Delta A+B F)^{T} P(A+\Delta A+B F)-P \\
& +L^{T}\left(B^{T} P B\right) L+L^{T} B^{T} P+P B L+4\left(A-A_{D}+\Delta A\right)^{T} P \\
& \times\left(A-A_{D}+\Delta A\right)<-\eta I .
\end{aligned}
$$

Letting $\bar{P}=P^{-1}$, it can be shown that

$$
\begin{aligned}
& \bar{P}(A+\Delta A+B F)^{T} \bar{P}^{-1}(A+\Delta A+B F) \bar{P}-\bar{P}+\bar{P} L^{T}\left(B^{T} \bar{P}^{-1} B\right) L \\
& \times \bar{P}+\bar{P} L^{T} B^{T}+B L \bar{P}+4 \bar{P}\left(A-A_{D}+\Delta A\right)^{T} \bar{P}^{-1} \\
& \times\left(A-A_{D}+\Delta A\right) \bar{P}<-\eta \bar{P}^{2} .
\end{aligned}
$$

Using Lemma 2, it may be demonstrated that the inequality in (37) can be implied by (38), shown at the top of the next page, where $X=F \bar{P}, Y=L \bar{P}$ and $\bar{\eta}=\eta^{-1}$. With the help of Lemma 1, (38) can be sufficed by the LMI in (19).

Remark 3: Note that the control law in (15) is referred to as Lyapunov min-max controller, since it minimizes the Lyapunov function's difference, $\Delta V$, for the worst case of the uncertainty; see e.g. [23].

Remark 4: Consider the following so-called linear controller,

$$
u(k)=-(S B)^{-1} S A_{D} x(k) .
$$

This linear controller can be used in the overall system (8) with external disturbance, and can guarantee the boundedness of the system state, in this case the ultimate bound on the closed-loop system state is as in (34) with $\gamma=4\|S B\|\|f\|^{2}$.

\section{VAriable Structure Controller CONSIDERATIONS}

As discussed in section II, in the literature, it is argued that the discontinuous part of the sliding control input can be detrimental to performance [3]. However, this claim is only true for the balanced uncertainties and/or disturbances whose the biggest frequency is close to the sampling rate of the discrete-time system. In simpler terms, when the sampling rate of the discrete signal processing system is big enough compared to the maximum component frequency of exogenous disturbance $f(k)$, the claim of [3] is not true. It has been shown in [10] that the term $\mathscr{F}^{+}=\operatorname{col}\left(\mathscr{F}_{1}^{+}, \cdots, \mathscr{F}_{h}^{+}\right)$ (see (12) and (13)) can be used to compensate the nonzero mean of the unbalanced disturbances. It can easily be realized that in the case of using $\mathscr{F}^{+}$the maximum estimation error is $\mathscr{F}^{c}$. Letting

$$
\vartheta(k)=\mathscr{F}^{+},
$$




$$
\left[\begin{array}{ccccc}
-\bar{P}+Y^{T} B^{T}+B Y & {[(A+\Delta A) \bar{P}+B X]^{T}} & Y^{T} B^{T} & 2 \bar{P}\left(A-A_{D}+\Delta A\right)^{T} & \bar{P} \\
(A+\Delta A) \bar{P}+B X & -\bar{P} & 0 & 0 & 0 \\
B Y & 0 & -\bar{P} & 0 & 0 \\
2\left(A-A_{D}+\Delta A\right) \bar{P} & 0 & 0 & -\bar{P} & 0 \\
\bar{P} & 0 & 0 & 0 & -\bar{\eta} I
\end{array}\right]<0
$$

the controller in (15) can be defined as the following one,

$$
u(k)=-(S B)^{-1} S A_{D} x(k)-\mathscr{F}^{+} .
$$

Also, the ultimate bound on the system state is as in (34) with $\gamma=4\|S B\|\left\|\mathscr{F}^{c}\right\|^{2}$, by setting $\tau=1$ in (16).

Remark 5: With a quick glimpse into the literature, it can be found that a frequently used candidate for the component $\vartheta(k)$ has the general form of:

$$
\vartheta(k)=\psi+v \operatorname{sgn}\left(\sigma_{x}(k)\right),
$$

where $\psi$ and $v$ are known parameters. For instance, in [24], with ignoring the bounds of $S \Delta A x(k)$ (see Remark 2), $\psi$ and $v$ are assumed to be some constants involving the bounds of $f(k)$, similar to $\mathscr{F}^{+}$and $\mathscr{F}^{-}$. Hence, the controller in (15) can be set in a similar manner as in [24] to be as

$$
u(k)=-(S B)^{-1} S A_{D} x(k)-\mathscr{F}^{+}-\mathscr{F}^{-} \operatorname{sgn}\left(\sigma_{x}(k)\right) .
$$

Regardless of different approaches used to design the parameters of this nonlinear function, it should be emphasized that the term $\operatorname{sgn}\left(\sigma_{x}(k)\right)$ is not an appropriate function to determine the position of the disturbance relative to its mean value either in the physical meaning or in the theoretical sense. Using the controller containing $\vartheta(k)$ as in (42) will lead state trajectories to chatter around the switching surface with amplitude dependent on the lower bound of the term (42) and with the frequency equal to the sampling rate; see [10].

\section{Simulation Results}

Consider system (8) with $h=2$ and

$$
\begin{aligned}
& A_{11}=\left[\begin{array}{ccc}
0.5 & 0.2 & 0.04 \\
-0.01 & 1.3 & 0.12 \\
-0.16 & -1.05 & 0.3
\end{array}\right], B_{1}=\left[\begin{array}{cc}
0.0038 & 1.00 \\
0.0810 & 0.10 \\
0.10 & 2
\end{array}\right] \text {, } \\
& A_{22}=\left[\begin{array}{cc}
0.4 & 0.9 \\
-1.1 & 0.2
\end{array}\right], B_{2}=\left[\begin{array}{c}
0.02 \\
1.5
\end{array}\right] \text {, } \\
& A_{12}=\left[\begin{array}{cc}
0.05 & -0.1 \\
-0.1 & 0.0 \\
-0.02 & 0.2
\end{array}\right], A_{21}=\left[\begin{array}{ccc}
-0.23 & 0.15 & 0.1 \\
0 & 0.1 & 0.2
\end{array}\right] \\
& M=\left[\begin{array}{lllll}
0.15 & 0.08 & 0.2 & -0.05 & 0.2
\end{array}\right]^{T} \text {, } \\
& N=\left[\begin{array}{lllll}
0.23 & 0.15 & -0.25 & -0.18 & -0.22
\end{array}\right] \text {, } \\
& R(k)=0.3 \sin (k) \text {, } \\
& f_{1}(k)=\left[\begin{array}{l}
0.1 \\
0.1
\end{array}\right] \sin \left(\frac{k}{5}\right), f_{2}(k)=0.1 \sin \left(\frac{k}{5}\right) \text {. }
\end{aligned}
$$
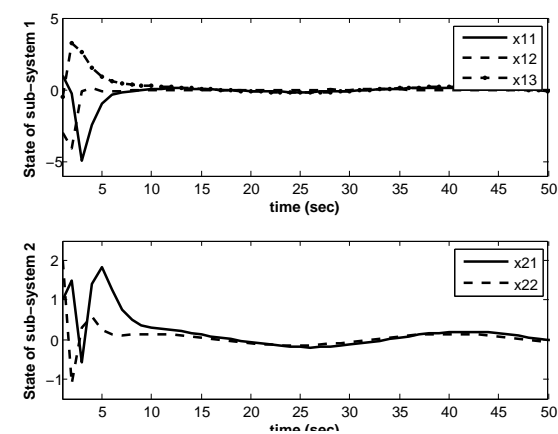

Fig. 1. Trajectories of the state using the linear controller

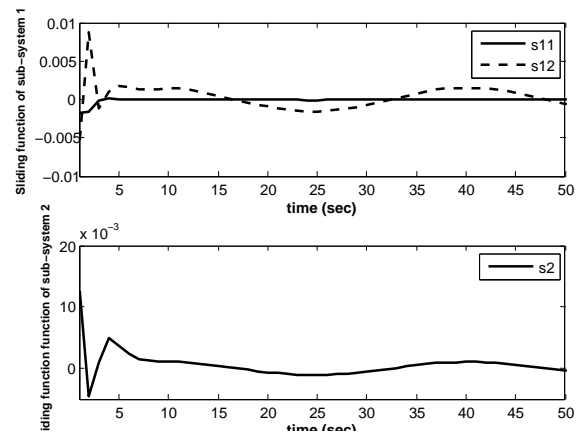

Fig. 2. Trajectories of the sliding function using the linear controller

Note that the open-loop sub-systems are unstable. Solving the LMI (19) gives the following results:

$$
\begin{aligned}
\bar{P}_{1} & =\left[\begin{array}{ccc}
292.1376 & -86.9891 & 51.3692 \\
-86.9891 & 152.3270 & 14.4219 \\
51.3692 & 14.4219 & 338.9188
\end{array}\right], \\
\bar{P}_{2} & =\left[\begin{array}{cc}
405.5726 & -82.0062 \\
-82.0062 & 278.9241
\end{array}\right], \\
\bar{\eta} & =789.2056 I_{5}, \varepsilon=310.7873 .
\end{aligned}
$$

Hence, using $P=\bar{P}^{-1}$ in the control law (39), the results of applying this linear controller to the overall system (8) are shown in Figs 1-2. Note that since the exogenous disturbance is balanced, $\mathscr{F}^{+}=\left[\begin{array}{ccc}0 & 0 & 0\end{array}\right]^{T}$ and thus the controller in (41) is same as (39). Here, the initial state is assumed to be $x(0)=\left[\begin{array}{lllll}1 & -3 & -0.5 & 1 & 2\end{array}\right]^{T}$. It can be seen that the states are ultimately bounded and also during the sliding motion the states are within a band of sliding surface $\sigma_{x}(k)=$ 0 .

Once again, we apply the controller in (43) to the overall system (8) using $\mathscr{F}^{-}=0.1 I_{3}$. The results are demonstrated in Figs 3-4. As seen, this controller leads the state trajectories 

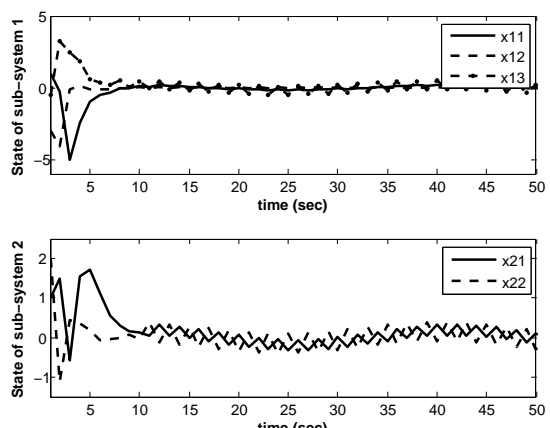

Fig. 3. Trajectories of the state using the controller (43)

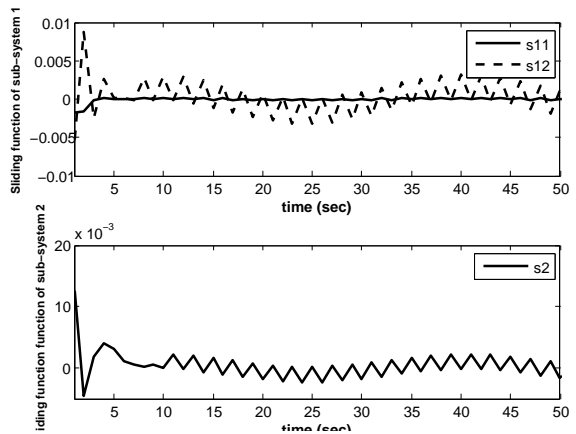

Fig. 4. Trajectories of the sliding function using the controller (43)

to chatter around the switching surface.

\section{Conclusions}

In this paper, using interconnections' information, we have used an LMI framework to design the decentralized sliding surface which can tolerate both interconnection influences and subsystems uncertainties. Besides, in order to have an entirely decentralized sliding surface, we have imposed the block-diagonal structure on the LMI variable. Using the obtained decentralized sliding surface, a linear decentralized DSMC has been proposed. Also, the boundedness of the state and sliding function of the underlying overall closedloop system is analyzed and the ultimate bound on the system state and the sliding function is derived. Moreover, the issue of variable structure decentralized controller has been discussed in this paper. The illustrative examples have shown the efficiency of the proposed robust decentralized DSMC.

\section{REFERENCES}

[1] C. Milosavljević, General conditions for the existence of a quasisliding mode on the switching hyperplane in discrete variable structure systems, Automation and Remote Control, vol. 3, 1985, pp 36-44.

[2] A. Bartoszewicz, Discrete-time quasi-sliding-mode control strategies, IEEE Transactions on Industrial Electronics, vol. 45, pp. 633-637, 1998.

[3] N. O. Lai, C. Edwards and S. K. Spurgeon, Discrete output feedback sliding-mode control with integral action, Int. J. Robust Nonlinear Control, vol. 16, pp. 21-43, 2006.

[4] S. Qu, X. Xia, and J. Zhang, Dynamics of Discrete-time Sliding Mode Control Uncertain System with a Disturbance Compensator, IEEE Transactions on Industrial Electronics, vol. 61, no. 7, pp. 35023510, 2014.
[5] Q. Xu, Enhanced discrete-time sliding mode strategy with application to piezoelectric actuator control, IET Control Theory and Applications, vol. 7, Iss. 18, pp. 2153-2163, 2013.

[6] W. Gao, Y. Wang, and A. Homaifa, Discrete-time variable structure control system, IEEE Trans. Ind. Electron, vol. 42, pp. 117-122, 1995

[7] A. J. Koshkouei, A. S. I. Zinober, Sliding mode control of discretetime systems, Journal of Dynamic Systems, Measurement, and Control, vol. 122, pp. 793-802, 2000.

[8] S. Z. Sapturk, Y. Istefanopulous, O. Kaynak, On the stability of discrete-time sliding mode control systems, IEEE Transactions on Automatic Control, vol. 32, pp. 930-932, 1987.

[9] S. K. Spurgeon, Hyperplane design techniques for discrete-time variable structure control systems, International Journal of Control vol. 55(2), pp. 445-456, 1992.

[10] S. Hui and S. H. Zak, On discrete-time variable structure sliding mode control, Systems and Control Letters, vol. 38, pp. 283-288, 1999.

[11] G. Monsees, Discrete-time sliding mode control, Ph.D. Thesis, Delft University of Technology, The Netherlands, 2002.

[12] X. G. Yan, C. Edwards, and S. K. Spurgeon, Decentralized robust sliding mode control for a class of nonlinear interconnected systems by static output feedback, Automatica, vol. 40, pp. 613-620, 2004.

[13] X. G. Yan, S. K. Spurgeon, and C. Edwards, Decentralised sliding mode control for nonminimum phase interconnected systems based on a reduced-order compensator, Automatica, vol. 42, pp. 1821-1828, 2006.

[14] L. Bakule, Decentralized control: An overview, Annual Reviews in Control, vol. 32, pp. 87-98, 2008

[15] V. A. Ugrinovskii, I. R. Petersen, A. V. Savkin, E. Y. Ugrinovskaya, Decentralized state-feedback stabilization and robust control of uncertain large-scale systems with integrally constrained interconnections, Systems and Control Letters, vol. 40, no. 2, pp. 107-119, 2000.

[16] A. I. Zečević, D. D. Śiljak, Global low-rank enhancement of decentralized control for large-scale systems, IEEE Transactions on Automatic Control, vol. 50, no. 5, pp. 740-744, 2005.

[17] D. T. Gavel, D. D. Śiljak, Decentralized adaptive control: structural conditions for stability, IEEE Transactions on Automatic Control, vol. 34, no. 4, pp. 413-426, 1989.

[18] D.D. Siljak, Decentralized Control of Complex Systems. Academic Press: San Diego, CA, 1991.

[19] W. J. Wang, Y. H. Chen, Decentralized robust control design with insufficient number of controllers, International Journal of Control, vol. 65, pp. 1015-1030, 1996.

[20] S. Boyd, L. E. Ghaoui, E. Feron, and V. Balakrishnan, Linear Matrix Inequalities in System and Control Theory, Society for Industrial and Applied Mathematics, Philadelphia, 1994.

[21] I. R. Peterson, A stabilization algorithm for a class of uncertain linear systems, Systems Control Lett. vol. 8 pp. 351357, 1987.

[22] L. Li, V. A. Ugrinovskiia, and R. Orsi, Decentralized robust control of uncertain Markov jump parameter systems via output feedback, Automatica, vol. 43, pp. 1932-1944, 2007.

[23] N. Sharav-Scapiro, Z. J. Palmor, and A. Steinberg, Output Stabilizing Robust Control for Discrete Uncertain Systems, Automatica, vol. 34, no. 6, pp. 731-739, 1998

[24] Y. Niu and D. W. C. Ho, Design of Sliding Mode Control Subject to Packet Losses, IEEE Transactions on Automatic Control, vol. 55, no. 11 , pp. 2623-2628, 2010.

[25] S. Govindaswamy, S. K. Spurgeon, and T. Floquet, Discrete-time output feedback sliding-mode control design for uncertain systems using linear matrix inequalities, International Journal of Control, vol. 84, no. 5, pp. 916930, 2011.

[26] H. K. Khalil, Nonlinear Systems, 3rd Edition, Prentice Hall, New York, 2002.

[27] W. Su, S. V. Drakunov and Ü. Özgüner, An $O\left(T^{2}\right)$ boundary layer in sliding mode for sampled-data systems, IEEE Transactions on Automatic Control, vol. 45(3), pp. 482-485, 2000.

[28] A. Qureshi and M. A. Abido, Decentralized discrete-time quasisliding mode control of uncertain linear interconnected systems, International Journal of Control, Automation and Systems, vol. 12(2), pp. 349-357, 2014

[29] M. S. Mahmoud and A. Qureshi, Decentralized sliding-mode outputfeedback control of interconnected discrete-delay systems, Automatica, vol. 48(5), pp. 808-814, 2012. 